\title{
Correlation analysis between the parameters of contrast-enhanced ultrasonography in evaluating cervical cancer metastasis and expression of E-cadherin
}

\author{
XIAOLAN LV, MIN HOU and XIAOJING DUAN \\ Department of Ultrasonography, Affiliated Hospital of Hebei University, Baoding, Hebei 071000, P.R. China
}

Received June 23, 2017; Accepted August 8, 2017

DOI: $10.3892 / 01.2017 .6785$

\begin{abstract}
The study aims to investigate the correlation between the parameters of contrast-enhanced ultrasonography in evaluating cervical cancer metastasis and expression of E-cadherin. All 120 patients with cervical cancer underwent contrast-enhanced ultrasonography. According to the results of postoperative pathological examination, patients were divided into distant metastasis group (group A), lymph node metastasis without distant metastasis group (group B) and no metastasis group (group C). Expression of E-cadherin in cervical cancer tissues was detected by enzyme-linked immunosorbent assay (ELISA). Correlations between the parameters of contrast-enhanced ultrasonography in evaluating cervical cancer metastasis and expression of E-cadherin were analyzed by Pearson's correlation analysis. Comparison of parameters of contrast-enhanced ultrasonography showed that, the baseline intensity of group A was $11.9 \pm 2.2 \mathrm{~dB}$, which was significantly lower than that of group B and C. Baseline intensity of group B was significantly lower than that of group C $(13.0 \pm 2.4$ vs. $15.3 \pm 3.6 \mathrm{~dB})$, significant differences were found among three groups $(\mathrm{P}<0.05)$. The sensitivity and specificity of the use of enhanced intensity $\sim 83.7 \mathrm{~dB}$ in evaluating tumor metastasis of patients with cervical cancer were 82.42 and $79.32 \%$, respectively. Expression level of E-cadherin protein in group A was $0.030 \pm 0.003 \mathrm{ng} / \mathrm{ml}$, which was significantly lower than that in group $\mathrm{B}$ and $\mathrm{C}(\mathrm{P}<0.05)$, expression level of E-cadherin protein in group A was significantly lower than that in group C $(0.037 \pm 0.007$ vs. $0.045 \pm 0.012 \mathrm{ng} / \mathrm{ml})$, significant differences in the expression level of E-cadherin protein were found among the three groups of cervical cancer patients $(\mathrm{P}<0.05)$. Pearson's correlation analysis showed that there was a positive correlation between the baseline intensity of contrast-enhanced ultrasonography and the expression level of E-cadherin $(\mathrm{P}<0.05)$, while there was a negative correlation
\end{abstract}

Correspondence to: Dr Min Hou, Department of Ultrasonography, Affiliated Hospital of Hebei University, 212 Yuhuadong Road, Baoding, Hebei 071000, P.R. China

E-mail: huk4a6@163.com

Key words: cervical cancer, contrast-enhanced ultrasonography, tumor metastasis, E-cadherin, enhanced intensity between the enhanced intensity of contrast-enhanced ultrasonography and the expression level of E-cadherin $(\mathrm{P}<0.05)$. Contrast-enhanced ultrasonography can be used to determine the tumor metastasis of cervical cancer patients, in addition, the combined use of contrast-enhanced ultrasonography and E-cadherin protein expression can significantly improve the diagnosis and treatment of cervical cancer.

\section{Introduction}

The incidence of cervical cancer is relative high among all malignant tumors in women. Accurate staging and early diagnosis play a key role in treatment and prognosis of cervical cancer. Cytology examination and related specialized examination are still the main methods used in the diagnosis of cervical cancer. However, these methods cannot be used to accurately determine the scope of tumor invasion (1). Ultrasonography plays a certain role in diagnosis and staging of cervical cancer. However, the function of ultrasonography is limited for patients with small lesions or without obvious morphological changes in cervix (2). Contrast-enhanced ultrasonography can be used to dynamically observe the characteristics of microcirculation blood perfusion in tissues, so this method can be used to effectively detect malignant tumors (3). Recent studies have shown that cell adhesion molecules play important roles in the onset and development of cancers. As a main type of cell adhesion molecule (4),E-cadherin is highly accumulated in epithelial cell of mature tissue. E-cadherin can participate in the transmission and exchange of information between cells and regulate the formation and development of embryonic tissues, E-cadherin can also maintain the polarity and integrity of the morphology of epithelial cells, and regulate adhesion aggregation between epithelial cells and the differentiation of epithelial cells (5). Lymph node metastasis can significantly affect the prognosis of cervical cancer. Therefore, the determination of lymph node metastasis has significant effect on the staged operation of patients with cervical cancer. In this study, patients with cervical cancer were examined by contrast-enhanced ultrasonography. Expression of E-cadherin in cervical cancer tissues of patients with different metastatic conditions was measured by enzyme-linked immunosorbent assay (ELISA). In addition, the correlation between the parameters of contrast-enhanced ultrasonography in evaluating cervical cancer metastasis and expression of E-cadherin were also explored. 


\section{Materials and methods}

General information. In total 120 patients with cervical cancer were selected from January 2015 to December 2016. i) Inclusion criteria: cervical cancer patients aged 45 to 65 years. ii) Exclusion criteria: patients with hepatitis virus and human immunodeficiency virus infection; patients with autoimmune diseases; patients with other types of malignant tumor; patients with severe cardiovascular and cerebrovascular diseases; patients with psychiatric disorders. iii) Causes of loss of contact and response measures: expected causes: patients quit the test, patients transferred to another hospital; response measures: add new participants at the ratio of 1:1 according to the inclusion and exclusion criteria. iv) Medical ethics issues: patients or their family members signed written informed consent, the safety of patients was ensured according to the relevant principles of clinical guidelines, and privacy (medical record) of patients were protected. v) Double-blind clinical experiments: researchers were divided into four groups, group 1 was responsible for the selection and grouping of patients, group 2 was responsible for the treatment, group 3 was responsible for the observation and data collection, and group 4 was responsible for statistical analysis and manuscript writing. The grouping is confidential to the patients, and the four groups of researchers were confidential to each other. This study was approved by the Ethics Committee of Affiliated Hospital of Hebei University. Signed written informed consents were obtained from the patients and/or guardians.

\section{Research methods}

Grouping. According to the results of postoperative pathological examination, 120 patients with cervical cancer were divided into distant metastasis group (group A), lymph node metastasis without distant metastasis group (group B) and no metastasis group (group C).

Contrast-enhanced ultrasonography. Contrast-enhanced ultrasonography was performed using a real-time threedimensional color Doppler Voluson E8 Ultrasonic Diagnostic Instrument (GE Healthcare, Cleveland, OH, USA). The probe frequency is $3.5 \mathrm{MHz}$ and the mechanical index is 0.11 . Routine abdominal or vaginal ultrasound examination was performed for all the patients to determine tumor size, location, shape and number. The transabdominal acoustic contrast agent SonoVue (Bracco, Courcouronnes, France) was used. Saline $(5 \mathrm{ml})$ was injected into the bottle containing SonoVue and mixed. Then SonoVue suspension $(2.0 \mathrm{ml})$ was injected through elbow vein, and $5 \mathrm{ml}$ of saline was then used. The built-in timer of the ultrasonic instrument was started. Changes in echo intensity of lesions and the process of contrast agent perfusion were observed, and the dynamic images were stored and quantitatively analyzed.

Quantitative analysis of ultrasound images. The built-in acoustic quantitative time-strength curve analysis software was used to draw the curve. Freehand method was used to cover the whole area of solid tumors. With the normal uterine muscle as a control, automatic marking method was used to obtain relevant quantitative parameters including baseline intensity, peak intensity, arrival time, time to peak, enhances intensity and perfusion time were calculated. Enhanced intensity (peak intensity-baseline intensity, perfusion time) time to peak-arrival
Table I. Clinicopathological parameters of cervical cancer patients.

Clinicopathological parameter Cases (n)

Types of tissue

Squamous cell carcinoma 93

Adenocarcinoma

Metastasis

Distant metastasis $\quad 28$

Lymph node metastasis $\quad 43$

No metastasis $\quad 49$

Pathological staging

Stage I

Stage II 38

Stage III

time. The ROC curve was drawn and the area below the curve was calculated. The sensitivity and specificity of different truncation points were calculated to estimate the ideal value of enhanced intensity in determining lymph node metastasis.

ELISA to detect the expression of E-cadherin in cervical cancer tissue. Human E-cadherin assay kit used in this study is from ImmunoWay Biotechnology Co. (Plano, TX, USA). The specific steps are as follows: i) blood was extracted from patients and transferred to coagulation tubes, followed by centrifugation $(2,650 \mathrm{xg})$ for $10 \mathrm{~min}$ to collect the supernatant, which was transferred to EP tubes and stored at $-80^{\circ} \mathrm{C}$; ii) The standard was diluted according to the instructions of kit and three repeat wells were set for each concentration; iii) Standard well, sample well and blank control well were set, and $100 \mu \mathrm{l}$ sample was added into each well, the plate was covered and incubated at $37^{\circ} \mathrm{C}$ for $2.5 \mathrm{~h}$; iv) $300 \mu \mathrm{l}$ of washing solution was added into each well, and washing was performed 4 times; v) $100 \mu \mathrm{l}$ of biotin-labeled antibody was added to each well and incubated for $1 \mathrm{~h}$ at room temperature; vi) step 4 was repeated; vii) $100 \mu 1$ of enzyme conjugate working solution was added into each well, the plate was covered and incubated at room temperature for $45 \mathrm{~min}$; viii) Step 4 was repeated; ix) $100 \mu \mathrm{l}$ of color development reagent was added into each well and incubated for 10-20 min at room temperature in the dark. x) $100 \mu \mathrm{l}$ of stop solution was added into each well and the absorbance at $450 \mathrm{~nm}$ was measured using a microplate reader.

Statistical analysis. All data were analyzed by SPSS 20.0 statistical analysis software (IBM, Armonk, NY, USA). Data were expressed as mean \pm standard deviation, and single-factor analysis of variance was performed, the comparison among multiple groups, and LSD t-test was performed for the comparisons between two groups, $\mathrm{P}<0.05$ was considered to be statistically significant.

\section{Results}

Clinicopathological parameters of cervical cancer patients. In this study, 120 patients with cervical cancer, including 93 patients with squamous cell carcinoma and 27 patients with adenocarcinoma were involved. Distant metastasis was found 
Table II. Comparison of contrast-enhanced ultrasonography parameters among three groups.

\begin{tabular}{|c|c|c|c|c|c|}
\hline Items & Group A $(n=28)$ & Group B $(n=43)$ & Group C (n=49) & F-value & P-value \\
\hline Baseline intensity (dB) & $11.9 \pm 2.2$ & $13.0 \pm 2.4^{\mathrm{a}}$ & $15.3 \pm 3.6^{\mathrm{a}, \mathrm{b}}$ & 15.367 & $<0.05$ \\
\hline Peak intensity (dB) & $95.8 \pm 9.3$ & $93.8 \pm 8.1$ & $91.3 \pm 9.5$ & 0.935 & $>0.05$ \\
\hline Enhanced intensity (dB) & $85.9 \pm 6.1$ & $84.3 \pm 6.6$ & $78.9 \pm 6.2$ & 0.881 & $>0.05$ \\
\hline Arrival time (sec) & $17.5 \pm 3.6$ & $17.0 \pm 3.4$ & $16.4 \pm 4.4$ & 0.827 & $>0.05$ \\
\hline Time to peak (sec) & $27.3 \pm 5.0$ & $27.0 \pm 5.3$ & $26.5 \pm 5.7$ & 0.904 & $>0.05$ \\
\hline Perfusion time (sec) & $10.2 \pm 2.4$ & $10.8 \pm 2.0$ & $11.2 \pm 1.8$ & 0.752 & $>0.05$ \\
\hline
\end{tabular}

${ }^{\mathrm{a} C o m p a r e d ~ w i t h ~ g r o u p ~} \mathrm{~A}, \mathrm{P}<0.05$; ${ }^{\mathrm{b}}$ compared with group $\mathrm{B}, \mathrm{P}<0.05$.

Table III. Comparison of sensitivity, specificity and area under ROC curve between different cut points of enhanced intensity.

\begin{tabular}{lccc}
\hline $\begin{array}{l}\text { Cut points } \\
\text { of enhanced } \\
\text { intensity (dB) }\end{array}$ & $\begin{array}{c}\text { Sensitivity } \\
(\%)\end{array}$ & $\begin{array}{c}\text { Specificity } \\
(\%)\end{array}$ & $\begin{array}{c}\text { Area } \\
\text { under } \\
\text { the curve }\end{array}$ \\
\hline 73.1 & 100 & 37.82 & 0.788 \\
83.7 & 82.42 & 79.32 & 0.809 \\
85.9 & 62.81 & 80.52 & 0.787 \\
86.0 & 60.53 & 80.51 & 0.776 \\
86.2 & 58.11 & 81.83 & 0.738 \\
86.8 & 47.60 & 100 & 0.725 \\
\hline
\end{tabular}

in 28 cases, lymph node metastasis without distant metastasis was found in 43 cases, and 49 cases showed no metastasis. Results of pathological staging showed that the number of patients with stage I, II and III were 31,38 and 51, respectively (Table I).

Comparison of contrast-enhanced ultrasonography parameters among three groups. Comparison of parameters of contrast-enhanced ultrasonography showed that, the baseline intensity of group A was $11.9 \pm 2.2 \mathrm{~dB}$, which was significantly lower than that of group B and C. Baseline intensity of group B was significantly lower than that of group C $(13.0 \pm 2.4$ vs. $15.3 \pm 3.6 \mathrm{~dB})$, significant differences were found among the three groups $(\mathrm{P}<0.05)$. The $\mathrm{P}$-value of the comparison of enhanced intensity among three groups was 0.052 , which is close to 0.05 . No significant differences in peak intensity, arrival time, time to peak and perfusion time were found among groups $(\mathrm{P}>0.05)$ (Table II).

Comparison of sensitivity, specificity and area under ROC curve between different cut points of enhanced intensity. In this study, ROC curve was drawn and the area under the curve was calculated to evaluate the sensitivity and specificity of different cut points of enhanced intensity in evaluating tumor metastasis of cervical cancer patients. Results showed that the sensitivity and specificity of the use of enhanced intensity $\sim 83.7 \mathrm{~dB}$ in evaluating tumor metastasis of patients with cervical cancer were 82.42 and $79.32 \%$, respectively, and the area under ROC curve was 0.809 (Table III and Fig. 1).

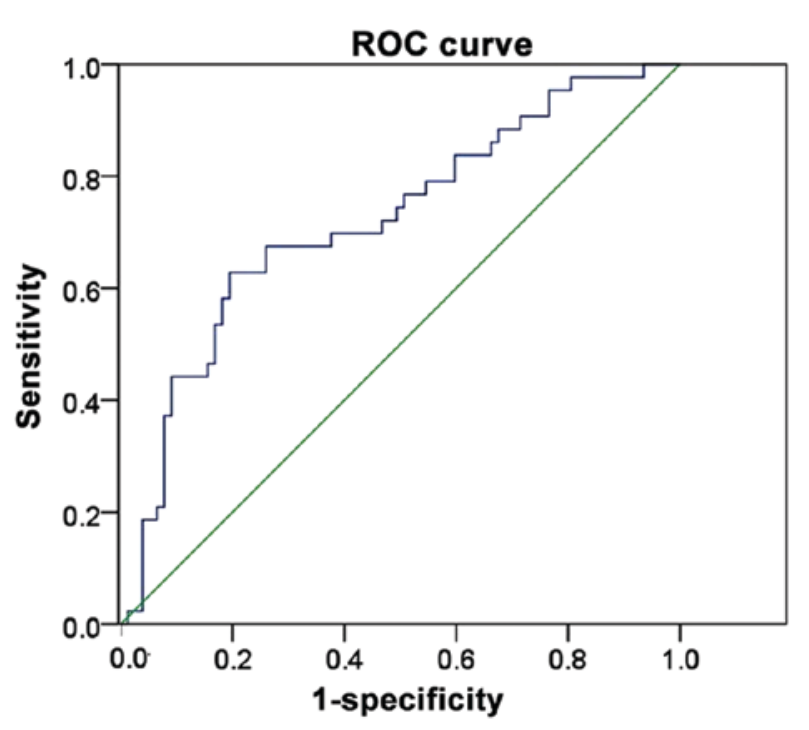

Figure 1. ROC curve analysis of the use of enhanced intensity $\sim 83.7 \mathrm{~dB}$ in evaluating tumor metastasis of patients with cervical cancer. The sensitivity and specificity of the use of enhanced intensity $\sim 83.7 \mathrm{~dB}$ in evaluating tumor metastasis of patients with cervical cancer were 82.42 and $79.32 \%$, respectively, and the area under ROC curve was 0.809 .

Expression of E-cadherin in cervical cancer tissues of patients in three groups. The expression of E-cadherin in cervical cancer tissues was detected by ELISA. Results showed that the expression level of E-cadherin protein in group A was $0.030 \pm 0.003 \mathrm{ng} / \mathrm{ml}$, which was significantly lower than that of group $\mathrm{B}$ and $\mathrm{C}(\mathrm{P}<0.05)$, and the expression level of $\mathrm{E}$-cadherin in group $B$ was significantly lower than that in group $C$ $(0.037 \pm 0.007$ vs. $0.045 \pm 0.012 \mathrm{ng} / \mathrm{ml})$, significant differences were found among groups $(\mathrm{P}<0.05)($ Table IV).

Correlations between the parameters of contrast-enhanced ultrasonography in evaluating cervical cancer metastasis and expression of E-cadherin. Correlations between the parameters of contrast-enhanced ultrasonography in evaluating cervical cancer metastasis and expression of E-cadherin were analyzed by Pearson's correlation analysis. Results showed that the baseline intensity of contrast-enhanced ultrasonography was positively correlated with the expression level of E-cadherin $(\mathrm{P}<0.05)$, while enhanced intensity of contrast-enhanced ultrasonography was negatively correlated with the expression level of E-cadherin $(\mathrm{P}<0.05)$ (Table V). 
Table IV. Comparison of expression levels of E-cadherin in cervical cancer tissues of patients in three groups.

\begin{tabular}{lcc}
\hline Groups & $\mathrm{n}$ & Level of E-cadherin $(\mathrm{ng} / \mathrm{ml})$ \\
\hline Group A & 28 & $0.030 \pm 0.003$ \\
Group B & 43 & $0.037 \pm 0.007^{\mathrm{a}}$ \\
Group C & 49 & $0.045 \pm 0.012^{\mathrm{a}, \mathrm{b}}$ \\
F-value & - & 23.148 \\
P-value & - & $<0.05$ \\
\hline
\end{tabular}

${ }^{\mathrm{a} C}$ Compared with group $\mathrm{A}, \mathrm{P}<0.05$; ${ }^{\mathrm{b}}$ compared with group $\mathrm{B}, \mathrm{P}<0.05$.

\begin{abstract}
Correlations between the parameters of contrast-enhanced ultrasonography in evaluating cervical cancer metastasis and clinicopathological parameters of patients. Correlations between the parameters of contrast-enhanced ultrasonography in evaluating cervical cancer metastasis and clinicopathological parameters of patients were performed by Pearson's correlation analysis. Results showed that baseline intensity and enhanced intensity were closely correlated with the tumor metastasis and pathological stages of patients. No significant correlation was found between parameters of contrast-enhanced ultrasonography and tissue types $(\mathrm{P}>0.05)$ (Table VI).
\end{abstract}

\section{Discussion}

The incidence of cervical cancer is relatively high among all malignant tumors in women. Biological characteristics of and staging of the tumors usually determine the selection of treatment strategies, take cervical cancer as an example, although studies have shown that abnormal expression of some cytokines can be used as indicators for the diagnosis of cervical cancer (6), the tumor size and scope of infiltration are still the key factors that determine the treatment and prognosis of cervical cancer. Cytology examination and related specialized examination are still the main methods used for the diagnosis of cervical cancer, so the scope of infiltration cannot be determined, let alone the metastasis, causing difficulties in diagnosis $(7,8)$. Ultrasound provides new insight for the staging and diagnosis of cervical cancer, and the accuracy of diagnosis was increased significantly with the use of ultrasound (9).
Table V. Correlations between the parameters of contrastenhanced ultrasonography in evaluating cervical cancer metastasis and expression levels of E-cadherin.

\begin{tabular}{lcc}
\hline $\begin{array}{l}\text { Parameters of } \\
\text { contrast-enhanced } \\
\text { ultrasonography }\end{array}$ & \multicolumn{2}{c}{ Expression levels of E-cadherin } \\
\cline { 2 - 3 } & rs & P-value \\
\hline Baseline intensity & 0.657 & 0.007 \\
Peak intensity & -0.164 & 0.235 \\
Enhanced intensity & -0.526 & 0.001 \\
Arrival time & -0.102 & 0.742 \\
Time to peak & -0.023 & 0.303 \\
Perfusion time & 0.171 & 0.869 \\
\hline
\end{tabular}

Insufficient blood supply is common for normal lymph nodes, while blood supply can be significantly improved with the existing of lymph node metastasis, so the conditions of blood supply of lymph nodes detected by ultrasound can be used to determine the lymph node metastasis (10). Intestinal gas interference and abdominal fat attenuation and other adverse conditions can affect the examination of abdominal, pelvic lymph nodes. In addition, color Doppler ultrasound is affected by the resolution of the instrument, operator's experience, sampling angle and parameter adjustment and other factors, so this technique is not sensitive to low-speed blood flow and cannot be used to fully understand the internal blood supply to lymph nodes. Therefore, conditions of blood supply to abdominal, pelvic lymph nodes detected by conventional ultrasound examination cannot be used to accurately determine the lymph node metastasis (11). Contrast-enhanced ultrasonography is a new imaging technique that can be used to dynamically and clearly observe microvascular, especially tumor blood vessels, by injecting contrast agents. Specific diagnosis of lesions can be made based on the patterns of contrast agent perfusion, such as speed and intensity. In this study, the baseline intensity of group A was $11.9 \pm 2.2 \mathrm{~dB}$, which was significantly lower than that of group $\mathrm{B}$ and $\mathrm{C}$. Baseline intensity of group B was significantly lower than that of group $C(13.0 \pm 2.4$ vs. $15.3 \pm 3.6 \mathrm{~dB})$, significant differences were found among three groups $(\mathrm{P}<0.05)$. The $\mathrm{P}$-value of the comparison of enhanced intensity among three groups

Table VI. Correlations between the parameters of contrast-enhanced ultrasonography in evaluating cervical cancer metastasis and clinicopathological parameters of patients.

\begin{tabular}{|c|c|c|c|c|c|c|}
\hline \multirow{2}{*}{$\begin{array}{l}\text { Parameters of } \\
\text { contrast-enhanced } \\
\text { ultrasonography }\end{array}$} & \multicolumn{2}{|c|}{ Tissue type } & \multicolumn{2}{|c|}{ Tumor metastasis } & \multicolumn{2}{|c|}{ Pathological stage } \\
\hline & rs & P-value & rs & P-value & rs & P-value \\
\hline Baseline intensity & 0.733 & 0.125 & 0.782 & 0.005 & 0.679 & 0.012 \\
\hline Peak intensity & 0.421 & 0.336 & 0.347 & 0.213 & 0.412 & 0.116 \\
\hline Enhanced intensity & 0.706 & 0.148 & 0.623 & 0.011 & 0.653 & 0.028 \\
\hline Arrival time & 0.112 & 0.657 & 0.135 & 0.654 & 0.388 & 0.235 \\
\hline Time to peak & 0.234 & 0.326 & 0.149 & 0.661 & 0.109 & 0.719 \\
\hline Perfusion time & 0.128 & 0.817 & 0.112 & 0.732 & 0.364 & 0.349 \\
\hline
\end{tabular}


was 0.052 , which is close to 0.05 . The data suggest that differences in the parameters of contrast-enhanced ultrasonography can be used to determine the status of lymph node metastasis. The quality of imaging can be affected by patient's body mass index, ultrasonic attenuation and the depth of lesions (12), the specificity and sensitivity of different cut points of enhanced intensity in determining tumor metastasis were compared. The initial intensity with contrast agent perfusion was the baseline intensity. Baseline intensity can be affected by scope of liquefactive necrosis and degree of fibrosis, so baseline intensity can be used to differentiation degrees and biological characteristics of tumors (13). The maximum intensity is the peak intensity. Peak intensity includes baseline intensity. So, the difference between peak intensity and the baseline strength is the enhanced intensity. Enhanced intensity is a normalized value and the differences of enhanced intensity between individuals were not big. So enhanced intensity can be used to more clearly understand the conditions of blood perfusion and more accurately determine metastasis of cervical cancer (14). Studies have confirmed that microvessel density can significantly affect the enhanced intensity of contrast-enhanced ultrasonography. As an accurate tumor angiogenesis index, microvessel density can significantly affect the onset and development (15). Microvessel density can also significantly affect lymph node metastasis, biological behavior and the degree of differentiation of tumors. One of the important causes of tumor lymph node metastasis is intravascular angiogenesis (16). In this study, the ROC curve was drawn and the area under the curve was calculated to evaluate the sensitivity and specificity of different cut-off points of enhanced intensity in predicting tumor lymph node metastasis. Results showed that the sensitivity and specificity of the use of enhanced intensity $\sim 83.7 \mathrm{~dB}$ in evaluating tumor metastasis of patients with cervical cancer were 82.42 and $79.32 \%$, respectively. In addition, Pearson's correlation analysis showed that baseline intensity and enhanced intensity were closely correlated with the tumor metastasis and pathological stages of patients.

E-cadherin is highly accumulated in epithelial cells of mature tissue and early stage embryo tissue. As a kind of transmembrane glycoprotein, E-cadherin can mediate adhesion between epithelial cells. As one of the tumor suppressor proteins, loss of E-cadherin expression can lead to the development and distant metastasis of tumors (17). E-cadherin inactivation is critical in the development of cervical cancer. Inactivation of E-cadherin can induce the occurrence of EMT and the activation of Wnt signaling pathway. Decreased E-cadherin expression can cause migration and invasion of tumor cells (18). Compared with wild-type cell line, the migration ability of E-cadherin mutant cell lines was significantly increased, indicating that cell migration can be affected by the inactivation of E-cadherin (19). In this study, the expression of E-cadherin in cervical cancer tissues was detected by ELISA. The results showed that the expression of E-cadherin protein in patients with distant metastasis was significantly lower than that in patients only with lymph node metastasis and patients without metastasis. Expression level of E-cadherin in patients only with lymph node metastasis was significantly higher than that in patients without metastasis, significant differences in the expression level of E-cadherin protein were found among three groups. This finding is consistent with previous studies (20), indicating that adhesion between cervical cancer cells can be reduced by the reduced expression of E-cadherin, leading to the failure of tissue in inhibiting metastasis, further causing the diffuse growth and distant metastasis of cervical cancer cells. So, the observation of the changes in expression level of E-cadherin can play a role in the diagnosis of cervical cancer.

In conclusion, quantitative analysis of the results of contrast-enhanced ultrasonography can play a certain role in the determination of cervical cancer metastasis. The combined use of contrast-enhanced ultrasonography and E-cadherin expression can significantly improve the diagnosis and treatment of cervical cancer.

\section{References}

1. Park JW, Park DM, Choi BK, Kwon BS, Seong JK, Green JE, Kim DY and Kim HK: Establishment and characterization of metastatic gastric cancer cell lines from murine gastric adenocarcinoma lacking Smad4, p53, and E-cadherin. Mol Carcinog 54: 1521-1527, 2015.

2. Huang X, Qian Y, Wu H, Xie X, Zhou Q, Wang Y, Kuang W, Shen L, Li K, Su J, et al: Aberrant expression of osteopontin and E-cadherin indicates radiation resistance and poor prognosis for patients with cervical carcinoma. J Histochem Cytochem 63: 88-98, 2015.

3. Liu Y, Chen XG and Liang CZ: Expressions of E-cadherin and $\mathrm{N}$-cadherin in prostate cancer and their implications. Zhonghua Nan Ke Xue 20: 781-786, 2014 (In Chinese).

4. Slowinska-Klencka D, Sporny S, Stasikowska-Kanicka O, Popowicz B and Klencki M: E-cadherin expression is more associated with histopathological type of thyroid cancer than with the metastatic potential of tumors. Folia Histochem Cytobiol 50: 519-526, 2012.

5. Dellaportas D, Koureas A, Contis J, Lykoudis PM, Vraka I, Psychogios D, Kondi-Pafiti A and Voros DK: Contrast-enhanced color Doppler ultrasonography for preoperative evaluation of sentinel lymph node in breast cancer patients. Breast Care (Basel) 10: 331-335, 2015.

6. Biedka M, Makarewicz R, Marszałek A, Sir J, Kardymowicz H and Goralewska A: Labeling of microvessel density, lymphatic vessel density and potential role of proangiogenic and lymphangiogenic factors as a predictive/prognostic factors after radiotherapy in patients with cervical cancer. Eur J Gynaecol Oncol 33: 399-405, 2012.

7. Shiyan L, Pintong H, Zongmin W, Fuguang H, Zhiqiang Z, Yan Y and Cosgrove D: The relationship between enhanced intensity and microvessel density of gastric carcinoma using double contrast-enhanced ultrasonography. Ultrasound Med Biol 35: 1086-1091, 2009.

8. Liu Y, Ye Z, Sun H and Bai R: Grading of uterine cervical cancer by using the ADC difference value and its correlation with microvascular density and vascular endothelial growth factor. Eur Radiol 23: 757-765, 2013.

9. Nakamura K, Joja I, Nagasaka T, Haruma T and Hiramatsu Y: Maximum standardized lymph node uptake value could be an important predictor of recurrence and survival in patients with cervical cancer. Eur J Obstet Gynecol Reprod Biol 173: 77-82, 2014.

10. Kamrava M: Potential role of ultrasound imaging in interstitial image based cervical cancer brachytherapy. J Contemp Brachytherapy 6: 223-230, 2014.

11. Zaridah S: A review of cervical cancer research in Malaysia. Med J Malaysia 69 (Suppl A): 33-41, 2014.

12. Benckert C, Thelen A, Cramer T, Weichert W, Gaebelein G, Gessner R and Jonas S: Impact of microvessel density on lymph node metastasis and survival after curative resection of pancreatic cancer. Surg Today 42: 169-176, 2012.

13. Jiang J, Shang X, Zhang H, Ma W, Xu Y, Zhou Q, Gao Y, Yu S and Qi Y: Correlation between maximum intensity and microvessel density for differentiation of malignant from benign thyroid nodules on contrast-enhanced sonography. J Ultrasound Med 33: 1257-1263, 2014. 
14. Tang MX, Mulvana H, Gauthier T, Lim AK, Cosgrove DO, Eckersley RJ and Stride E: Quantitative contrast-enhanced ultrasound imaging: A review of sources of variability. Interface Focus 1: 520-539, 2011.

15. Li B, Shi H, Wang F, Hong D, Lv W, Xie X and Cheng X: Expression of E-, P- and N-cadherin and its clinical significance in cervical squamous cell carcinoma and precancerous lesions. PLoS One 11: e0155910, 2016

16. Peng J, Qi S, Wang P, Li W, Song L, Liu C and Li F: Meta-analysis of downregulated E-cadherin as a poor prognostic biomarker for cervical cancer. Future Oncol 24: 102-104, 2015.

17. Wagih HM,El-Ageery SM and Alghaithy AA: A study of RUNX3, E-cadherin and $\beta$-catenin in CagA-positive Helicobacter pylori associated chronic gastritis in Saudi patients. Eur Rev Med Pharmacol Sci 19: 1416-1429, 2015.
18. Myong NH: Loss of E-cadherin and acquisition of vimentin in epithelial-mesenchymal transition are noble indicators of uterine cervix cancer progression. Korean J Pathol 46: 341-348, 2012.

19. Cheng Y, Zhou Y, Jiang W, Yang X, Zhu J, Feng D, Wei Y, Li M, Yao F, Hu W, et al: Significance of E-cadherin, $\beta$-catenin, and vimentin expression as postoperative prognosis indicators in cervical squamous cell carcinoma. Hum Pathol 43: 1213-1220, 2012.

20. Do TV, Kubba LA, Du H, Sturgis CD and Woodruff TK: Transforming growth factor-beta1, transforming growth factorbeta2, and transforming growth factor-beta3 enhance ovarian cancer metastatic potential by inducing a Smad3-dependent epithelial-to-mesenchymal transition. Mol Cancer Res 6: 695-705, 2008. 